\title{
Stop smoking for the next generation. (Mini-commentary on BJOG-19-1709.R1)
}

\author{
Tao Duan ${ }^{1}$ and Weilin Wang ${ }^{2}$ \\ ${ }^{1}$ Shanghai First Maternity and Infant Hospital of Tongji University \\ ${ }^{2}$ Shanghai First Maternity and Infant Hospital
}

May 6, 2020

Pregnancy provides optimal opportunities for health promotion and disease prevention. Smoking before and during pregnancy is one of the largest modifiable risk factors for a range of adverse pregnancy outcomes. Concerns over the risks of smoking motivate many pregnant smokers to quit. However, most of their partners continue to smoke throughout pregnancy. More than one-third (35\%) of men in the world smoke, and just over $6 \%$ of women do (Ritchie et al. Published online at OurWorldInData.org 2020). The impact of paternal smoking, second-hand or third-hand smoking on the perinatal health is largely overlooked. Given the vast majority of studies focusing on the pregnant smokers, less is known about paternal smoking on their future children.

In this paper (Zhou et al. BJOG 2020), using a large national prepregnancy registry database, Zhou et al. found that paternal smoking may be associated with birth defects such as congenital heart diseases, limb abnormalities and neural tube defects in the offspring. The result was consistent with previous retrospective studies, but a medium to large effect size was seen. All of the odd ratios exceeded 2.5, with one even over 20 (congenital heart diseases $\mathrm{OR}=2.51,95 \% \mathrm{CI} 1.04-6.05$; limb abnormalities $\mathrm{OR}=20.64$ 95\% CI 6.46-68.02; digestive tract anomalies $\mathrm{OR}=3.6795 \% \mathrm{CI} 1.44-9.37$; neural tube defect $\mathrm{OR}=4.87,95 \% \mathrm{CI} 1.66-14.28)$. Such difference might be partially explained by potential confounding factors. For example, the extent of tobacco exposure, in the National Birth Defects Prevention Study (Malik et al. Pediatrics 2008; 121: e810), compared with light smoking exposure ( $<14$ cigarettes per day), heavy smoking women ([?]25 cigarettes per day) were more likely to have infants with septal defects (OR 2.06; 95\%CI: 1.20-3.54), suggesting a dose-dependent relationship between tobacco exposure and birth defects.

In addition, a reduced risk of birth defects after changing smoking behaviors was found in Zhou's paper. It is no surprise that paternal smoking cessation could improve the adverse outcomes. Limited evidences address whether there is a critical window period by which smoking must be stopped to prevent subsequent adverse perinatal outcomes. Results from a retrospective case-control study indicated that the risk of fetal congenital heart diseases increased as women smoked during the first trimester (Sullivan et al. J Pediatr; 2015; 166:978). In a prospective cohort study (McCowan et al. BMJ 2009; 338: b1081), the authors concluded that stopping smoking early in pregnancy, and certainly by 15-16 weeks' gestation, may minimize the adverse effects of smoking on late pregnancy complications and should be an important goal for pregnant smokers. Yet, such critical time for paternal smoking cessation remains unknown.

Tobacco smoke is also a human germ cell mutagen. It is estimated that with even a modest $25 \%$ increase in sperm mutation frequency caused by smoke-exposure, for each generation across the global population there will be millions of smoking-induced de novo mutations transmitted from father to offspring (Beal et 
al. Mutation Research;2017; 773:26).

Therefore, it is strongly recommended that women and men should immediately stop smoking in advance of reproduction.

No disclosures: A completed disclosure of interest form is available to view online as supporting information. 